\title{
Energy Content of Colliding Plane Waves using Approximate Noether Symmetries
}

\author{
M. Sharif *and Saira Waheed ${ }^{\dagger}$ \\ Department of Mathematics, University of the Punjab, \\ Quaid-e-Azam Campus, Lahore-54590, Pakistan.
}

\begin{abstract}
This paper is devoted to study the energy content of colliding plane waves using approximate Noether symmetries. For this purpose, we use approximate Lie symmetry method of Lagrangian for differential equations. We formulate the first-order perturbed Lagrangian for colliding plane electromagnetic and gravitational waves. It is shown that in both cases, there does not exist non-trivial first-order approximate symmetry generator.
\end{abstract}

Keywords: Colliding plane waves; Approximate symmetries; Conserved quantities.

\section{Introduction}

For the description of a physical system, the conserved quantities like energy, linear and angular momentum etc. are of great importance. Being a non-conserved quantity, energy and its localization is the most prominent and fundamental issue in general relativity. For gravitational waves (the ripples produced by accelerating mass in the fabric of the spacetime which

*msharif.math@pu.edu.pk

†sairawaheed_50@yahoo.com 
propagates through the space at the speed of light [1]), this problem is of particular interest. The gravitational waves, by definition, have zero stressenergy tensor. Thus the existence of these waves was questioned. However, the theory of general relativity indicates the existence of gravitational waves as solutions of the Einstein's field equations [2]. In fact this problem arises because energy is not well-defined in general relativity. The problem for plane gravitational waves was resolved by Ehlers and Kundt [3], Pirani [4] and Weber and Wheeler [5] by considering a sphere of test particles in the path of the waves. They showed that these particles acquired a constant momentum from the waves. Qadir and Sharif [6] presented an operational procedure, embodying the same principle, to show that gravitational waves impart a momentum.

To find a satisfactory solution of this challenging problem, enormous efforts have been made. The pseudo-tensors which are firstly introduced by Einstein provide a mean to determine global energy-momentum conservation. This attracted many others physicists to develop different prescriptions e.g. [7]-[13]. Being a combination of $T_{a}^{b}$ and a pseudo-tensor $t_{a}^{b}$ (as it explicates the energy and momentum density of the gravitational field), none of these non-tensorial complexes are proved to be unambiguous.

Recently, an alternative approach, i.e., concept of approximate symmetry, is used to define energy of gravitational waves. There have been a number of definitions of approximate symmetry and different methods [14, 15] are available to find these symmetries. Using this concept, a measure of the extent of the break-down of time-translational symmetry was presented [16]-[18]. This yields the so-called an almost symmetric space and almost Killing vector (the vector field corresponding to almost symmetric space) [19]. However, each of these attempts have their own drawbacks.

Noether symmetries also known as symmetries of the Lagrangian, provide a systematic treatment for the solution of DE arising in many practical problems [20]. Noether and Lie symmetries have many important applications like the linearization of the non-linear equation, the reduction of the order of ordinary differential equations (ODEs) as well as the number of independent variables of partial differential equations (PDEs) etc. The double reduction of DEs and the existence of conservation laws or first integrals (Noether invariants) are other interesting features of the Noether symmetries [21]. The more symmetries an equation possesses, the more easier will be its integration. The field equations that provide a basis for general theory of relativity (GR) are highly non-linear coupled PDEs. Finding an exact 
solution to these field equations is one of the major problems in GR. The Noether symmetries approach has been proved to be fruitful in this regard due to their wide range of applications in the field of DEs [22, 23]. It is worth mentioning here that most of physically interesting solutions to the field equations, available in literature, possess some kind of symmetry [24].

The Killing vectors (KVs) form a subalgebra of Noether symmetries which further form a subalgebra of Lie point symmetries. By investigating Noether symmetries and the corresponding conservation laws for some static models, it was shown that Noether symmetries yield some non-trivial conservation laws that are different from KVs [22]. The perturbed Lagrangian yields the approximate symmetries and the approximate conserved quantities as constants of motion. By contracting the first order non-trivial approximate symmetry with the momentum 4-vector, one can obtain energy non-conservation due to time variation [25]. Since Noether theorem leads to the fact that each continuous symmetry generator of the Lagrangian corresponds to a conserved quantity. Therefore, in order to define energy, it is very useful to utilize the concept of time symmetry (due to the fact that energy conservation comes from the time translational invariance).

In going from Minkowski spacetime (flat) to non-flat spacetimes, some of the conservation laws are lost. Using the connection between spacetime isometries and its related differential equations (geodesics) [26, 27] for different spacetimes, the lost conservation laws are recovered and the corresponding energy re-scaling factors are obtained [28]-[31]. We have used this procedure to discuss energy in the stringy charged black hole solutions [32] and Bardeen model [33]. In a recent paper [25], energy contents of pp and cylindrical gravitational waves have been discussed by using the same procedure. In this paper, we extend this procedure to colliding plane electromagnetic and gravitational waves to check the existence of conserved quantity.

The plan of this paper is as follows. In the next section, we review the mathematical framework for exact and approximate symmetry methods for the Lagrangian of differential equations (DEs). Sections 3 and 4 contain approximate Noether symmetries of colliding plane electromagnetic and gravitational waves respectively. The last section provides summary and results. 


\section{Mathematical Formulation}

Noether's theorem was proved in 1915 and published in 1918 by Emmy Noether. This theorem describes the connections between continuous symmetries of a physical system and their corresponding conserved quantities [34]. By the analysis of various transformations, Noether's theorem provides amazing insights into any general theory. This theorem provides information regarding the conservation laws. If the results of a physical experiment are independent of any change in position (homogeneity in space) and time, then the formulated Lagrangian is symmetric under continuous spacetime translations and this theorem leads to laws of energy and linear momentum conservation [35, 36]. Similarly, if the physical experiment is independent of any change in the measurement of angle, then the physical system is rotationally symmetric and this theorem implies the conservation law of angular momentum.

Noether's theorem has a wide range of applications in theoretical physics and calculus of variation. Dissipative system is one of the examples of the systems which cannot be modeled with Lagrangian. Therefore, Noether's theorem is inapplicable to these systems and consequently their continuous symmetries provide no conservation laws. The original form of this theorem is valid for those Lagrangian which contain only the first-order derivatives. In many practical problems, DEs arise with a small term called the perturbation parameter which indicates a small error or correction. The purpose for introducing this small term is to examine the given DE in some certain limit. The Lie point symmetries of such DEs (perturbed) are of great importance. The application of Noether symmetry analysis to Lagrangian of a system provides those symmetries which directly yield our desired conserved quantities. In that case, one needs to use only the first-order prolongation of the symmetry generator. The procedure for calculating the symmetries of the Lagrangian [37, 38] is given as follows.

We take a vector field $\mathbf{X}$ as

$$
\mathbf{X}=\xi\left(s, x^{a}\right) \frac{\partial}{\partial s}+\eta^{b}\left(s, x^{a}\right) \frac{\partial}{\partial x^{b}}
$$

and its first-order prolongation is defined as

$$
\mathbf{X}^{[1]}=\mathbf{X}+\left(\eta_{, s}^{b}+\eta_{, a}^{b} \dot{x}^{a}-\xi_{, s} \dot{x}^{b}-\xi_{, a} \dot{x}^{a} \dot{x}^{b}\right) \frac{\partial}{\partial \dot{x}^{b}}, \quad(a, b=0,1,2,3) .
$$


Suppose a second-order ODE, i.e., Euler-Lagrange equation is given by

$$
\ddot{x}^{a}=g\left(s, x^{a}, \dot{x}^{a}\right) .
$$

The vector field $\mathbf{X}$ is said to be Noether point symmetry of the Lagrangian $L\left(s, x^{a}, \dot{x}^{a}\right)$ (corresponding to Eq.(21)), if there exists a function $A\left(s, x^{a}\right)$ such that the following condition is satisfied

$$
\mathbf{X}^{[1]} L+\left(D_{s} \xi\right) L=D_{s} A .
$$

Here $D_{s}$ is the total derivative operator given by

$$
D_{s}=\frac{\partial}{\partial s}+\dot{x}^{a} \frac{\partial}{\partial x^{a}}
$$

and $A$ is a gauge function.

The significance of Noether symmetries is given by the following theorem.

Theorem: Suppose $L\left(s, x^{a}, \dot{x}^{a}\right)$ be the Lagrangian corresponding to secondorder ODE given by Eq.(2) and $\mathbf{X}$ is its corresponding Noether point symmetry. Then the first integral of motion associated with the Noether point symmetry $\mathbf{X}$ is defined [39] as

$$
I=\xi L+\left(\eta^{a}-\dot{x}^{a} \xi\right) \frac{\partial L}{\partial \dot{x}^{a}}-A .
$$

Now we define approximate Noether symmetries for DEs. The first-order perturbed Lagrangian corresponding to the first-order perturbed DE

$$
\mathbf{E}=\mathbf{E}_{0}+\epsilon \mathbf{E}_{1}+O\left(\epsilon^{2}\right)
$$

is given by

$$
L\left(s, x^{a}, \dot{x}^{a}, \epsilon\right)=L_{0}\left(s, x^{a}, \dot{x}^{a}\right)+\epsilon L_{1}\left(s, x^{a}, \dot{x}^{a}\right)+O\left(\epsilon^{2}\right)
$$

such that the functional $\int_{V} L d s$ is invariant under the one-parameter group of transformations with approximate Lie symmetry generator

$$
\mathbf{X}=\mathbf{X}_{0}+\epsilon \mathbf{X}_{1}+O\left(\epsilon^{2}\right)
$$

and

$$
A=A_{0}+\epsilon A_{1}
$$


is the first-order perturbed gauge function. Also, here

$$
\mathbf{X}_{b}=\xi_{b} \frac{\partial}{\partial s}+\eta_{b}^{a} \frac{\partial}{\partial x^{a}} ; \quad a=0,1,2,3, b=0,1
$$

Then the conditions for calculating exact and first-order symmetries are given as follows

$$
\begin{aligned}
& \mathbf{X}_{0}^{[1]} L_{0}+\left(D_{s} \xi_{0}\right) L_{0}=D_{s} A_{0}, \\
& \mathbf{X}_{1}^{[1]} L_{0}+\mathbf{X}_{0}^{[1]} L_{1}+\left(D_{s} \xi_{1}\right) L_{0}+\left(D_{s} \xi_{0}\right) L_{1}=D_{s} A_{1},
\end{aligned}
$$

where $\mathbf{X}_{0}$ and $\mathbf{X}_{1}$ are the exact and first-order parts, respectively, of the symmetry generator $\mathbf{X}$. The perturbed Lagrangian always admits a symmetry $\epsilon \mathbf{X}_{0}$ called the trivial symmetry. If, for the symmetry generator $\mathbf{X}$ to exist with $\mathbf{X}_{0} \neq 0$ and $\mathbf{X}_{1} \neq k \mathbf{X}_{0}$, where $k$ is any arbitrary constant, then $\mathbf{X}$ is called the non-trivial symmetry. The corresponding exact and the first-order approximate parts of the first integral are given by

$$
\begin{aligned}
& I_{0}=\xi_{0} L_{0}+\left(\eta_{0}^{a}-\dot{x}^{a} \xi_{0}\right) \frac{\partial L_{0}}{\partial \dot{x}^{a}}-A_{0}, \\
& I_{1}=\xi_{0} L_{1}+\xi_{1} L_{0}+\left(\eta_{0}^{a}-\dot{x}^{a} \xi_{0}\right) \frac{\partial L_{1}}{\partial \dot{x}^{a}}+\left(\eta_{1}^{a}-\dot{x}^{a} \xi_{1}\right) \frac{\partial L_{0}}{\partial \dot{x}^{a}}-A_{1} .
\end{aligned}
$$

The first integral of motion $I=I_{0}+\epsilon I_{1}$ is said to be unstable if $I_{0}=0$; otherwise it is stable.

For further details, one can see the literature [39]-[41].

\subsection{Exact and Approximate Symmetries of the Collid- ing Plane Electromagnetic Waves}

Bell and Szekeres, in 1974, presented an exact solution of field equations due to the collision and the subsequent interaction of two electromagnetic waves [42]. They considered the case of two-step electromagnetic waves which collide on the flat background region. The spacetime is defined in terms of

four regions [43]. Under the coordinate transformations $u=\frac{t-z}{2}, v=\frac{t+z}{2}$ 
[44], the spacetimes in these four regions can be written as

$$
\begin{aligned}
& \operatorname{Region}(\mathbf{I})(t<z, t<-z): \\
& d s^{2}=\frac{d t^{2}}{2}-d x^{2}-d y^{2}-\frac{d z^{2}}{2},
\end{aligned}
$$

Region(II) $(t>z, t<-z)$ :

$$
d s^{2}=\frac{d t^{2}}{2}-\cos ^{2} a\left(\frac{t-z}{2}\right)\left(d x^{2}+d y^{2}\right)-\frac{d z^{2}}{2},
$$

$\operatorname{Region}(\mathrm{III})(t<z, t>-z)$ :

$d s^{2}=\frac{d t^{2}}{2}-\cos ^{2} b\left(\frac{t+z}{2}\right)\left(d x^{2}+d y^{2}\right)-\frac{d z^{2}}{2}$,

$\operatorname{Region}(\mathbf{I V})(t>z, t>-z)$ :

$$
d s^{2}=\frac{d t^{2}}{2}-\cos ^{2}(A t-B z) d x^{2}-\cos ^{2}(A z-B t) d y^{2}-\frac{d z^{2}}{2},
$$

where $A=\frac{a-b}{2}$ and $B=\frac{a+b}{2}$. The solution has a special property of being conformally flat, i.e., all components of the Weyl tensor vanish.

In order to discuss Noether symmetries for colliding plane electromagnetic waves, we define Lagrangian for the spacetime given by Eq.(14) as follows

$$
L=\frac{\dot{t}^{2}}{2}-\cos ^{2}(A t-B z) \dot{x}^{2}-\cos ^{2}(A z-B t) \dot{y}^{2}-\frac{\dot{z}^{2}}{2} .
$$

To evaluate exact Noether symmetries of this Lagrangian, we substitute values of the Lagrangian, the first-order prolongation of the symmetry generator (given by Eq.(1)) and the total derivative operator (given by Eq.(4) ) in Eq.(17). Then we compare coefficients of the coordinate derivatives and their 
products on both sides. In this way, we obtain the following set of DEs

$$
\begin{aligned}
& \xi_{, t}=0, \quad \xi_{, x}=0, \quad \xi_{, y}=0, \quad \xi_{, z}=0, \quad A_{, s}=0, \\
& \eta_{, s}^{0}=A_{, t}, \quad-2 \cos ^{2}(A t-B z) \eta_{, s}^{1}=A_{, x}, \\
& -2 \cos ^{2}(A z-B t) \eta_{, s}^{2}=A_{, y}, \quad-\eta_{, s}^{3}=A_{, z}, \\
& \eta_{, x}^{0}-2 \cos ^{2}(A t-B z) \eta_{, t}^{1}=0, \\
& \eta_{, y}^{0}-2 \cos ^{2}(A z-B t) \eta_{, t}^{2}=0, \quad \eta_{, z}^{0}-\eta_{, t}^{3}=0, \\
& \cos ^{2}(A t-B z) \eta_{, y}^{1}+\cos ^{2}(A z-B t) \eta_{, x}^{2}=0, \\
& \eta_{, x}^{3}+2 \cos ^{2}(A t-B z) \eta_{, z}^{1}=0, \quad 2 \eta_{, t}^{0}-\xi_{, s}=0, \\
& \eta_{, y}^{3}+2 \cos ^{2}(A z-B t) \eta_{, z}^{2}=0, \quad 2 \eta_{, z}^{3}-\xi_{, s}=0, \\
& 2 \eta_{, x}^{1}-\xi_{, s}-2(A-B) \tan (A t-B z)\left(\eta^{0}+\eta^{3}\right)=0, \\
& 2 \eta_{, y}^{2}-\xi_{, s}-2(A-B) \tan (A z-B t)\left(\eta^{0}+\eta^{3}\right)=0 .
\end{aligned}
$$

This is a system of 19 DEs which we have to solve for the five unknowns $\xi, \eta^{0}, \eta^{1}, \eta^{2}, \eta^{3}$ using back and forth substitutions (where each of these is a function of five variables $s, t, x, y, z$ ). The solution (exact symmetry generators) to the above set of DEs can be written as

$$
\mathbf{Y}_{0}=s \frac{\partial}{\partial t}-s \frac{\partial}{\partial z}, \quad \mathbf{Y}_{1}=\frac{\partial}{\partial x}, \quad \mathbf{Y}_{2}=\frac{\partial}{\partial y}
$$

It has been mentioned earlier that the electromagnetic waves collide with each other on the flat background region (i.e., Minkowski spacetime in Cartesian coordinates) and is represented by non-static spacetime (given by Eq.(14)). Therefore we define it as a perturbation over some static spacetime, i.e., spacetime representing the interaction region (region (IV)) as a perturbation over the flat Minkowski spacetime. The exact static spacetime is given by

$$
d s^{2}=\frac{d t^{2}}{2}-d x^{2}-d y^{2}-\frac{d z^{2}}{2}
$$

and the corresponding Lagrangian can be written as

$$
L=\frac{\dot{t}^{2}}{2}-\dot{x}^{2}-\dot{y}^{2}-\frac{\dot{z}^{2}}{2}
$$

We evaluate Noether symmetries of this Lagrangian by using Eq.(7). After substituting all the corresponding values, we compare coefficients of the 
coordinate derivatives and their products and obtain the following set of determining equations

$$
\begin{aligned}
& \xi_{, t}=0, \quad \xi_{, x}=0, \quad \xi_{, y}=0, \quad \xi_{, z}=0, \quad A_{, s}=0 \\
& \eta_{, s}^{0}=A_{, t}, \quad-2 \eta_{, s}^{1}=A_{, x}, \quad-\eta_{, s}^{3}=A_{, z}, \\
& -2 \eta_{, s}^{2}=A_{, y}, \quad \eta_{, x}^{0}-2 \eta_{, t}^{1}=0 \\
& \eta_{, y}^{0}-2 \eta_{, t}^{2}=0, \quad \eta_{, z}^{0}-\eta_{, t}^{3}=0, \quad \eta_{, y}^{1}+\eta_{, x}^{2}=0 \\
& \eta_{, x}^{3}+2 \eta_{, z}^{1}=0, \quad 2 \eta_{, t}^{0}-\xi_{, s}=0 \\
& \eta_{, y}^{3}+2 \eta_{, z}^{2}=0, \quad 2 \eta_{, z}^{3}-\xi_{, s}=0 \\
& 2 \eta_{, x}^{1}-\xi_{, s}=0, \quad 2 \eta_{, y}^{2}-\xi_{, s}=0 .
\end{aligned}
$$

The simultaneous solution for this set of DEs is given by

$$
\begin{aligned}
\xi & =\frac{c_{0} s^{2}}{2}+c_{1} s+c_{2} \\
\eta^{0} & =\frac{t\left(c_{0} s+c_{1}\right)}{2}+s c_{3}+2 x c_{5}+2 c_{7} y+c_{12} z+c_{13} \\
\eta^{1} & =\frac{x\left(c_{0} s+c_{1}\right)}{2}+s c_{4}+t c_{5}+c_{8} y+c_{14} z+c_{15} \\
\eta^{2} & =\frac{y\left(c_{0} s+c_{1}\right)}{2}+s c_{6}+t c_{7}+c_{16} z-x c_{8}+c_{17} \\
\eta^{3} & =\frac{z\left(c_{0} s+c_{1}\right)}{2}-s c_{9}+t c_{12}-2 x c_{14}-2 y c_{16}+c_{11} \\
A(t, x, y, z) & =c_{0}\left(\frac{t^{2}}{4}-\frac{x^{2}}{2}-\frac{y^{2}}{2}-\frac{z^{2}}{4}\right)+t c_{3}-2 x c_{4}-2 y c_{6}+c_{9} z+c_{10}
\end{aligned}
$$

where all c's are arbitrary constants of integration. Clearly this is a 17 dimensional algebra in which many symmetries are present which provide no conservation laws. In this algebra, 10 symmetries correspond to the generators which form the Poincare group and 7 provide the other significant generators which have been discussed in detail [30]. These symmetries can 
be re-arranged to the following form

$$
\begin{aligned}
\mathbf{Y}_{0} & =\frac{s t}{2} \frac{\partial}{\partial t}+\frac{s x}{2} \frac{\partial}{\partial x}+\frac{s y}{2} \frac{\partial}{\partial y}+\frac{s z}{2} \frac{\partial}{\partial z} \\
\mathbf{Y}_{1} & =\frac{Y_{0}}{s}, \mathbf{Y}_{2}=s \frac{\partial}{\partial t}, \mathbf{Y}_{3}=2 x \frac{\partial}{\partial t}+t \frac{\partial}{\partial x} \\
\mathbf{Y}_{4} & =s \frac{\partial}{\partial y}, \mathbf{Y}_{5}=\frac{\partial}{\partial x}, \quad \mathbf{Y}_{6}=2 y \frac{\partial}{\partial t}+t \frac{\partial}{\partial y} \\
\mathbf{Y}_{7} & =y \frac{\partial}{\partial x}-x \frac{\partial}{\partial y}, \mathbf{Y}_{8}=-s \frac{\partial}{\partial z}, \mathbf{Y}_{9}=\frac{\partial}{\partial z} \\
\mathbf{Y}_{10} & =z \frac{\partial}{\partial t}+t \frac{\partial}{\partial z}, \mathbf{Y}_{11}=\frac{\partial}{\partial t} \\
\mathbf{Y}_{12} & =z \frac{\partial}{\partial x}-2 x \frac{\partial}{\partial z}, \quad \mathbf{Y}_{13}=\frac{\partial}{\partial x}, \\
\mathbf{Y}_{14} & =z \frac{\partial}{\partial y}-2 y \frac{\partial}{\partial z}, \mathbf{Y}_{15}=\frac{\partial}{\partial y}, \mathbf{Y}_{16}=s \frac{\partial}{\partial x}
\end{aligned}
$$

In order to calculate conserved quantity by using the non-trivial firstorder Noether symmetry (if it exists), we define the perturbed spacetime as follows

$$
d s^{2}=\frac{d t^{2}}{2}-\left[1+\epsilon \cos ^{2}(A t-B z)\right] d x^{2}-\left[1+\epsilon \cos ^{2}(A z-B t)\right] d y^{2}-\frac{d z^{2}}{2} .
$$

(Basically it means that we are taking the spacetime of region (IV) as a perturbation over the spacetime of region $(\mathbf{I})$ ). The corresponding first-order perturbed Lagrangian is given by

$$
L=\frac{\dot{t}^{2}}{2}-\left[1+\epsilon \cos ^{2}(A t-B z)\right] \dot{x}^{2}-\left[1+\epsilon \cos ^{2}(A z-B t)\right] \dot{y}^{2}-\frac{\dot{z}^{2}}{2} .
$$

The first-order approximate symmetries of this Lagrangian is found by using Eq.(8). After substituting the Lagrangian and other corresponding values, we make comparison of the coefficients of coordinate derivatives and their 
product terms. Consequently we obtain the following set of DEs

$$
\begin{aligned}
& \xi_{, t}=0, \quad \xi_{, x}=0, \quad \xi_{, y}=0, \quad \xi_{, z}=0, \quad A_{, s}=0, \\
& \eta_{, s}^{0}=A_{, t}, \quad-2 \eta_{, s}^{1}-2 \cos ^{2}(A t-B z)\left[\frac{x c_{0}}{2}+c_{4}\right]=A_{, x}, \\
& -2 \eta_{, s}^{2}-\cos ^{2}(A z-B t)\left[\frac{y c_{0}}{2}+c_{6}\right]=A_{, y}, \quad-\eta_{, s}^{3}=A_{, z}, \\
& \eta_{, x}^{0}-2 \eta_{, t}^{1}-2 c_{5} \cos ^{2}(A t-B z)=0, \\
& \eta_{, y}^{0}-2 \eta_{, t}^{2}-2 c_{7} \cos ^{2}(A z-B t)=0, \quad \eta_{, z}^{0}-\eta_{, t}^{3}=0, \\
& \eta_{, y}^{1}+\eta_{, x}^{2}+c_{8}\left[\cos ^{2}(A t-B z)-\cos ^{2}(A z-B t)\right]=0, \\
& \eta_{, x}^{3}+2 \eta_{, z}^{1}+2 c_{14} \cos ^{2}(A t-B z)=0,2 \eta_{, t}^{0}-\xi_{, s}=0, \\
& \eta_{, y}^{3}+2 \eta_{, z}^{2}+2 c_{16} \cos ^{2}(A z-B t)=0, \quad 2 \eta_{, z}^{3}-\xi_{, s}=0, \\
& 2 \eta_{, x}^{1}-\xi_{, s}-2(A-B) \cos (A t-B z) \sin (A t-B z)\left[\frac{t\left(c_{0} s+c_{1}\right)}{2}\right. \\
& +\frac{z\left(c_{0} s+c_{1}\right)}{2}+s\left(c_{3}-c_{9}\right)+2 x\left(c_{5}-c_{14}\right)+2 y\left(c_{7}-c_{16}\right) \\
& \left.+c_{12}(t+z)+c_{13}+c_{11}\right]+\left(c_{0} s+c_{1}\right) \cos ^{2}(A t-B z)=0, \\
& 2 \eta_{, y}^{2}-\xi_{, s}-2(A-B) \cos (A z-B t) \sin (A z-B t)\left[\frac{t\left(c_{0} s+c_{1}\right)}{2}\right. \\
& +\frac{z\left(c_{0} s+c_{1}\right)}{2}+s\left(c_{3}-c_{9}\right)+2 x\left(c_{5}-c_{14}\right)+2 y\left(c_{7}-c_{16}\right) \\
& \left.+c_{12}(t+z)+c_{13}+c_{11}\right]-\left(c_{0} s+c_{1}\right) \cos ^{2}(A z-B t)=0=0 .
\end{aligned}
$$

In this system of DEs, 14 out of 17 constants corresponding to exact symmetry generators are present. We solve this system of DEs by back and forth substitutions and check whether there exist some non-trivial parts of the symmetry generators or not. Since all the constants corresponding to the exact symmetry generators given by Eqs.(36)-(40) disappear, therefore the system of DEs given in Eqs.(44)-(53) becomes homogeneous. Thus it yields symmetries of the static spacetime only.

\subsection{Exact and Approximate Symmetries of the Collid- ing Plane Gravitational Waves}

The spacetime representing the collision of two plane gravitational waves in Rosen form is given by

$$
d s^{2}=2 e^{-M} d u d v-e^{-U}\left(e^{V} d x^{2}+e^{-V} d y^{2}\right),
$$


where $U, V$ and $M$ are functions of the null coordinates $u$ and $v$. For the collision of such waves, one has the following division of the spacetime into four regions. The spacetimes in these four regions are given by [45]:

$\operatorname{Region}(\mathbf{I})(u<0, v<0)$ :

$$
d s^{2}=2 d u d v-d x^{2}-d y^{2}
$$

$\operatorname{Region}(\mathrm{II})(u>0, v<0)$ :

$d s^{2}=2(1 \pm u)^{\frac{\left(a^{2}-1\right)}{2}} d u d v-(1 \pm u)^{1-a} d x^{2}-(1 \pm u)^{1+a} d y^{2}$,

Region(III) $(u<0, v>0)$ :

$d s^{2}=2(1 \pm v)^{\frac{\left(a^{2}-1\right)}{2}} d u d v-(1 \pm v)^{1-a} d x^{2}-(1 \pm v)^{1+a} d y^{2}$,

Region(IV) $(u>0, v>0)$ :

$$
\begin{aligned}
& d s^{2}=2(1 \pm u \pm v)^{\frac{\left(a^{2}-1\right)}{2}} d u d v-(1 \pm u \pm v)^{1-a} d x^{2} \\
& -(1 \pm u \pm v)^{1+a} d y^{2}
\end{aligned}
$$

where $a$ is any arbitrary parameter. If one chooses $a=0$ and the negative signs only, then this solution is quite similar to the solution obtained by Szekeres and Khan and Penrose [46. Using the suitable coordinate transformations $u=\frac{t-z}{2}, v=\frac{t+z}{2}$ [4] and taking only the negative signs, we have the spacetime in region (IV) as follows

$$
d s^{2}=2(1-t)^{\frac{-1}{2}}\left(\frac{d t^{2}}{4}-\frac{d z^{2}}{4}\right)-(1-t)\left(d x^{2}+d y^{2}\right)
$$

To evaluate exact symmetries of the Lagrangian for this spacetime

$$
L=2(1-t)^{\frac{-1}{2}}\left(\frac{\dot{t^{2}}}{4}-\frac{\dot{z}^{2}}{4}\right)-(1-t)\left(\dot{x^{2}}+\dot{y^{2}}\right)
$$

we use Eq.(7) and substitute the values of first-order prolongation operator, the total derivative operator and the above defined Lagrangian in it. Then we compare the coefficients of the derivatives and their products, we obtain 
the following set of DEs

$$
\begin{aligned}
& \xi_{, t}=0, \quad \xi_{, x}=0, \quad \xi_{, y}=0, \quad \xi_{, z}=0, \quad A_{, s}=0, \\
& \eta_{, s}^{0} \frac{1}{\sqrt{1-t}}=A_{, t}, \quad-2(1-t) \eta_{, s}^{1}=A_{, x}, \\
& -2(1-t) \eta_{, s}^{2}=A_{, y}, \quad-\eta_{, s}^{3} \frac{1}{\sqrt{1-t}}=A_{, z}, \\
& \eta_{, x}^{0}-2(1-t)^{3 / 2} \eta_{, t}^{1}=0, \\
& \eta_{, y}^{0}-2(1-t)^{3 / 2} \eta_{, t}^{2}=0, \quad \eta_{, z}^{0}-\eta_{, t}^{3}=0, \\
& \eta_{, y}^{1}+\eta_{, x}^{2}=0, \quad \eta_{, x}^{3}+2(1-t)^{3 / 2} \eta_{, z}^{1}=0, \\
& 2(1-t)\left[2 \eta_{, t}^{0}-\xi_{, s}\right]+\eta^{0}=0, \quad \eta_{, y}^{3}+2(1-t)^{3 / 2} \eta_{, z}^{2}=0, \\
& 2(1-t)\left(2 \eta_{, z}^{3}-\xi_{, s}\right)-\eta^{0}=0, \\
& (1-t)\left[2 \eta_{, x}^{1}-\xi_{, s}\right]-\eta^{0}=0, \quad(1-t)\left[2 \eta_{, y}^{2}-\xi_{, s}\right]-\eta^{0}=0 .
\end{aligned}
$$

The simultaneous solution of this system of DEs turns out to be

$\eta^{0}=0, \quad \eta^{1}=b_{6} y+b_{7}, \quad \eta^{2}=-b_{6} x+b_{8}, \quad \eta^{3}=b_{5}, \quad \xi_{2}=b_{2}, \quad A=b_{4},(69)$

where all b's are constants, which show that the gauge function is constant. These can be re-arranged to the following form

$$
A=b_{0}, \quad \xi_{2}=c_{0}, \quad \mathbf{Y}_{1}=\frac{\partial}{\partial z}, \quad \mathbf{Y}_{2}=y \frac{\partial}{\partial x}-x \frac{\partial}{\partial y}, \quad \mathbf{Y}_{3}=\frac{\partial}{\partial x}, \quad \mathbf{Y}_{4}=\frac{\partial}{\partial y}
$$

In order to discuss the corresponding conserved quantity, we take Eq.(58) as a perturbation over background static spacetime in a similar pattern as we have done for colliding electromagnetic waves. The background static spacetime is the Minkowski spacetime in Cartesian coordinates given by Eq.(27) and its Noether symmetries are given by Eqs.(36)-(40). T evaluate the approximate Noether symmetries, the first-order perturbed spacetime for the collision of gravitational waves is given by

$$
d s^{2}=\left[1+\epsilon(1-t)^{\frac{-1}{2}}\right]\left(\frac{d t^{2}}{2}-\frac{d z^{2}}{2}\right)-[1+\epsilon(1-t)]\left(d x^{2}+d y^{2}\right) .
$$

The corresponding Lagrangian is formulated as

$$
L=\left[1+\epsilon(1-t)^{\frac{-1}{2}}\right]\left(\frac{\dot{t^{2}}}{2}-\frac{\dot{z^{2}}}{2}\right)-[1+\epsilon(1-t)]\left(\dot{x^{2}}+\dot{y^{2}}\right) .
$$


Using this Lagrangian and the corresponding values in Eq.(8), we obtain the following system of DEs

$$
\begin{aligned}
& \xi_{, t}=0, \xi_{, x}=0, \xi_{, y}=0, \xi_{, z}=0, A_{, s}=0 \\
& \eta_{, s}^{0}+\left(\frac{c_{0} t}{2}+c_{3}\right) \frac{1}{\sqrt{1-t}}=A_{, t}, \\
& -2 \eta_{, s}^{1}-2(1-t)\left(\frac{x c_{0}}{2}+c_{4}\right)=A_{, x}, \\
& -2 \eta_{, s}^{2}-2(1-t)\left(\frac{c_{0} y}{2}+c_{6}\right)=A_{, y} \\
& -\eta_{, s}^{3}-\frac{1}{\sqrt{1-t}}\left(\frac{c_{0} z}{2}-c_{9}\right)=A_{, z}, \\
& {\left[\eta_{, x}^{0}-2 \eta_{, t}^{1}\right]+\frac{2}{\sqrt{1-t}} c_{5}\left(1-(1-t)^{3 / 2}\right)=0,} \\
& {\left[\eta_{, y}^{0}-2 \eta_{, t}^{2}\right]+\frac{2}{(1-t)^{1 / 2}} c_{7}\left(1-(1-t)^{3 / 2}\right)=0,} \\
& \eta_{, z}^{0}-\eta_{, t}^{3}=0, \eta_{, y}^{1}+\eta_{, x}^{2}=0 \\
& {\left[\eta_{, x}^{3}+2 \eta_{, z}^{1}\right]-\frac{2 c_{14}}{\sqrt{1-t}}\left(1-(1-t)^{3 / 2}\right)=0} \\
& {\left[2 \eta_{, t}^{0}-\xi_{, s}\right]+\frac{1}{2(1-t)^{3 / 2}}\left[\frac{t}{2}\left(c_{0} s+c_{1}\right)+s c_{3}+2 x c_{5}+2 c_{7} y\right.} \\
& \left.+c_{12} z+c_{13}\right]=0 \text {, } \\
& \eta_{, y}^{3}+2 \eta_{, z}^{2}-\frac{2 c_{16}}{\sqrt{1-t}}\left(1-(1-t)^{3 / 2}\right)=0 \\
& \left(2 \eta_{, z}^{3}-\xi_{, s}\right)+\frac{1}{2(1-t)^{3 / 2}}\left[\frac{t}{2}\left(c_{0} s+c_{1}\right)+s c_{3}+2 x c_{5}+2 c_{7} y\right. \\
& \left.+c_{12} z+c_{13}\right]=0 \text {, } \\
& {\left[2 \eta_{, x}^{1}-\xi_{, s}\right]-\left(\frac{t}{2}\left(c_{0} s+c_{1}\right)+s c_{3}+2 x c_{5}+2 c_{7} y\right.} \\
& \left.+c_{12} z+c_{13}\right)=0 \\
& {\left[2 \eta_{, y}^{2}-\xi_{, s}\right]-\left(\frac{t}{2}\left(c_{0} s+c_{1}\right)+s c_{3}+2 x c_{5}+2 c_{7} y\right.} \\
& \left.+c_{12} z+c_{13}\right)=0 \text {. }
\end{aligned}
$$

In this system of 19 DEs, 12 out of 17 constants, corresponding to exact symmetry generators defined in Eqs.(36)-(40), appear. When we solve this system of DEs simultaneously then in the final solution all these constants 
disappear. Therefore it makes the system of equations homogeneous and no non-trivial symmetry generator exists.

\section{Summary and Discussion}

In this paper, we have discussed energy contents for the colliding plane waves. For this purpose, we have applied the slightly broken or approximate Lie symmetry methods for Lagrangian to colliding plane electromagnetic and gravitational waves. Since the contraction of non-trivial approximate Noether symmetry (corresponding to time) with the momentum 4-vector can be used to define energy imparted to test particles [25]. Therefore, we have defined the first-order perturbed Lagrangian for both these solutions and check whether there exists some non-trivial symmetry.

We have obtained a system of 19 DEs both for the colliding plane electromagnetic as well as colliding plane gravitational waves. For the colliding plane electromagnetic waves, 14 out of 17 constants whereas for colliding plane gravitational waves, 12 out of 17 constants corresponding to exact symmetry generators defined in the Eqs.(36)-(40) appear. When we solve these systems of DEs simultaneously, all these constants disappear in both cases. Thus, the resulting systems of DEs becomes homogeneous and are the same as that for the exact case (i.e., Minkowski spacetime in Cartesian coordinates). Consequently only the previous exact symmetry generators of static spacetime which correspond to 17 dimensional Lie algebra for Minkowski sapcetime in Cartesian coordinates are obtained. These symmetry generators provide the conservation laws for energy, linear momentum, angular and spin angular momentum and hence there does not exist any non-trivial symmetry.

Thus we conclude that for these first-order perturbed Lagrangian for colliding plane electromagnetic and gravitational waves, there does not exist the conserved quantity. It is quite similar to the case of pp and cylindrical gravitational waves [25] where no non-trivial symmetry generator exists and one cannot determine the conserved quantity. It is mentioned here that one cannot apply these methods to the case of electromagnetic waves as there is no self-interaction. However, it may give significant result about the selfdamping or enhancement of the gravitational waves if one applies this method to the system of geodesic equations for these spacetimes. 


\section{References}

[1] Schutz, B.: Gravity from the Ground Up (Cambridge University Press, 2003).

[2] Misner, C.W., Thorne, K.S. and Wheeler, J.A.: Gravitation (W.H. Freeman, San Francisco, 1973).

[3] Ehlers, J. and Kundt, W.: Gravitation: An Introduction to Current Research, ed. L. Witten (Wiley, New York, 1962) 49.

[4] Pirani, F.A.E.: Gravitation: An Introduction to Current Research, ed. L. Witten (Wiley, New York, 1962)199.

[5] Weber, J. and Wheeler, J.A.: Rev. Mod. Phy. 29(1957)509.

[6] Qadir, A. and Sharif, M.: Physics Letters A 167(1992)331.

[7] Møller, C.: The Theory of Relativity (Oxford University Press, 1957).

[8] Landau, L.D. and Lifshitz, E.M.: The Classical Theory of Fields (Pergamon Press, 1975).

[9] Papapetrou, A.: Proc. R. Irish. Acad. A52(1958)11.

[10] Weinberg, S.: Gravitation and Cosmology: Principles and Applications of General Theory of Relativity (John Wiley and Sons, 1972).

[11] Møller, C.: Ann. Phys. 12(1961)118.

[12] Bondi, H.: Nature 186(1960)535.

[13] Ashtekar, A. and Hansen, RO.: J. Math. Phys. 19(1978)1542.

[14] Baikov, V., Gazizov, R.K. and Ibragimov, N.H.: Mat. Sb. 136(1988)435 (English transl.: Math. USSR Sb. 64(1989)427).

[15] Fushchich, W.I. and Shtelen, W.M.: J. Phys. A.: Math. Gen. 22(1989)L887.

[16] Komar, A.: Phys. Rev. 127(1962)1411; 129(1963)1873.

[17] Matzner, R.: J. Math. Phys. 9(1968)1657; 1063. 
[18] Isaacson, R.A.: Phys. Rev. 166(1968)1263; 1272.

[19] York, J.W.: Ann. Inst. H. Poincare Sect. (N.S.) A21(1974)319.

[20] Hussain, I. and Feroz, T.: J. Geometry and Phys. 61(2011)658.

[21] Stephani, H.: Differential Equations, their Solution Using Symmetries (Cambridge University Press, 1989).

[22] Bokhari, A.H. et al.: Int. J. Theor. Phys. 45(2006)1063.

[23] Soh. C.W. and Mahomad, F.M.: Class. Quantum Grav. 16(1999)3553.

[24] Bokhari, A.H. and Kara, A.H.: Gen. Relativ. Grav. 39(2007)2053.

[25] Hussain, I., Mahomed, F.M. and Qadir, A.: Phys. Rev. D79(2009)125014.

[26] Feroze, T., Mahomed, F.M. and Qadir, A.: Nonlinear Dynam. 45(2006)65.

[27] Aminova, A.V.: Sb. Math. 186(1995)1711.

[28] Kara, A.H., Mahomed, F.M., and Qadir, A.: Nonlinear Dynamics $\mathbf{5 1}(2008) 183$.

[29] Hussain, I., Mahomed, F.M. and Qadir, A.: SIGMA 3(2007)115.

[30] Hussain, I., Mahomed, F.M. and Qadir, A.: Gen. Relativ. Grav. 41(2009)2399.

[31] Hussain, I.: Gen. Relativ. Gravit. (2010, to appear).

[32] Sharif, M. and Waheed, S.: Canadian J. Phys. 88(2010)833.

[33] Sharif, M. and Waheed, S.: Physica Scripta (2011, to appear).

[34] Pritchard, C.: The Changing Shape of Geometry: Celebrating a Century of Geometry and Geometry Teaching (Cambridge University Press, 2002).

[35] http://mathworld.wolfram.com/NoethersSymmetryTheorem.html.

[36] Hanc, J., Tuleja, S. and Hancova, M.: American J. Phys. 72(2004)428. 
[37] Ibragimov, N.H.: Elementary Lie Group Analysis and Ordinary Differential Equations (John Wiley and Sons, 1999).

[38] Feroze, T. and Kara, A.H.: Int. J. Nonlinear Mechanics 37(2002)275.

[39] Ovsiannikov, L.V.: Group Analysis of Differential Equations (Academic Press, 1980).

[40] Kara, A.H., Mahomed, F.M. and Unal, G.: Int. J. Theor. Phys. 38(1999)2389.

[41] Unal, G.: Phys. Lett. A269(2000)13.

[42] Bell, P. and Szekeres, P.: Gen. Relativ. Grav. 5(1974)275.

[43] Clarke, C.J.S. and Hayward, S.A.: Class. Quantum Grav. 6(1989)615.

[44] Sharif, M. and Nazir, K.: Braz. J. Phys. 38(2008)156.

[45] Stoyanov, B.J.: Phys. Rev. D20(1979)2469.

[46] Szekeres, P.: J. Math. Phys. 13(1972)286. 